\title{
Can Bioactive Compounds of Crocus sativus L. Influence the Metabolic Activity of Selected CYP Enzymes in the Rat?
}

\author{
G. DOVRTĚLOVÁ ${ }^{1,2}$, K. NOSKOVÁ ${ }^{1,2}$, J. JUŘICA ${ }^{1,2}$, M. TURJAP ${ }^{1,3}$, O. ZENDULKA ${ }^{1,2}$ \\ ${ }^{1}$ Department of Pharmacology, Faculty of Medicine, Masaryk University, Brno, Czech Republic, \\ ${ }^{2}$ CEITEC (Central European Institute of Technology) Masaryk University, Brno, Czech Republic, \\ ${ }^{3}$ Department of Clinical Pharmacy, University Hospital, Ostrava, Czech Republic
}

Received September 14, 2015

Accepted September 25, 2015

\begin{abstract}
Summary
Safranal and crocin are biologically active compounds isolated from Crocus sativus L., commonly known as saffron. Clinical trials confirm that saffron has antidepressant effect, thus being a potential valuable alternative in the treatment of depression. The aim of the present study was to determine, whether systemic administration of safranal and crocin can influence the metabolic activity of CYP3A, CYP2C11, CYP2B, and CYP2A in rat liver microsomes (RLM). The experiments were carried out on male Wistar albino rats intragastrically administered with safranal $(4,20$, and $100 \mathrm{mg} / \mathrm{kg} /$ day) or with intraperitoneal injections of crocin $(4,20$, and $100 \mathrm{mg} / \mathrm{kg} /$ day). Our results demonstrate the ability of safranal and crocin to increase the total protein content and to change the metabolic activity of several CYP enzymes assessed as CYP specific hydroxylations of testosterone in RLM. Crocin significantly decreased the metabolic activity of all selected CYP enzymes, while safranal significantly increased the metabolic activity of CYP2B, CYP2C11 and CYP3A enzymes. Therefore, both substances could increase the risk of interactions with co-administered substances metabolized by cytochrome P450 enzymes.
\end{abstract}

\section{Key words}

Crocin • Safranal • CYP • Rat liver microsomes

\section{Corresponding author}

O. Zendulka, Department of Pharmacology, Faculty of Medicine, Masaryk University, Kamenice 5, 62500 Brno, Czech Republic. E-mail: zendulka@med.muni.cz

\section{Introduction}

Crocus sativus L. is a perennial flowering plant and a member of the Iridaceae family. Saffron, the dried stigmas of Crocus sativus flowers, is a well-known spice. Chemical analysis of saffron extracts has revealed about 150 different compounds from which the most studied are crocin, safranal, and picrocrocin (Winterhalter and Straubinger 2000, Bathaie and Mousavi 2010). Crocin as a water-soluble carotenoid gives saffron its typical reddish or yellowish color. Safranal is a monoterpenic aldehyde responsible for saffron's characteristic odour, and the bitter taste of saffron is attributed to picrocrocin, which is also a precursor of safranal (Tarantilis et al. 1995, Melnyk et al. 2010). Saffron has not always been primarily a spice (for flavoring and coloring of food), but rather a medicinal plant, which was used in traditional medicine as e.g. sedative, anxiolytic, expectorant, aphrodisiac, antispasmodic, and for the treatment of premenstrual syndrome, asthma, or pain (Schmidt et al. 2007, Ríos et al. 1996).

Several food supplements containing saffron extracts are already available worldwide. The antidepressant effect of saffron extract was described in several double blind, randomized, placebo-controlled clinical trials (Moshiri et al. 2006, Akhondzadeh et al. 2005) and its activity was comparable to the effect of clinically used antidepressants, namely fluoxetine (Basti et al. 2007, Noorbala et al. 2005) and imipramine (Akhondzadeh et al. 2004). Thus, saffron may become a valuable alternative to classical antidepressants. Safranal and crocin have been intensively studied in the 
last few years also for other biological effects, such as antiproliferative, antioxidative, hypnosedative, etc. (Rezaee and Hosseinzadeh 2013, Alavizadeh and Hosseinzadeh 2014, Hosseinzadeh and Noraei 2009, Moshiri et al. 2014).

It was described that the metabolic activity of cytochrome P450, which is one of the most important enzymatic systems for xenobiotic biotransformation, is influenced by a large number of natural substances, including carotenoids. The modulation of CYP metabolic activity could lead to clinically relevant changes in plasma concentrations of concurrently administered drugs, and thus also to changes in their pharmacological properties. The knowledge of the influence of saffron or its compounds on CYP enzymes is lacking. Therefore, it is important to reveal whether the activity of CYP enzymes could be affected. The aim of the present study is to determine the effect of systemic administration of safranal and crocin on the metabolic activity of CYP2C11, CYP3A, CYP2B, and CYP2A, and the total protein and total cytochrome $\mathrm{P} 450$ (CYP) content in rat liver microsomes (RLM).

\section{Methods}

\section{Chemicals}

Crocin and safranal were purchased form SigmaAldrich (St. Louis, MO, USA). Other compounds used in the study were following: NADP, glucose-6-phosphate dehydrogenase, glucose-6-phosphate, $\mathrm{MgCl}_{2}$, EDTA, prednisone, testosterone, $\mathrm{KH}_{2} \mathrm{PO}_{4}, \mathrm{NA}_{2} \mathrm{HPO}_{4}$, sucrose, and $\mathrm{KCl}$, all of them provided by Sigma-Aldrich (St. Louis, MO, USA). The metabolites of testosterone, namely $2 \beta-, 2 \alpha-, 7 \alpha-, 6 \beta-, 16 \alpha-$, and 16 16 -hydroxytestosterone were purchased from Steraloids Inc. (Newport, RI, USA). Chemicals and organic solvents for HPLC analysis (acetonitrile, methanol, and dichloromethane) were provided by Lach-ner (Neratovice, CZ).

\section{Animal procedures}

The experiments were carried out on male Wistar albino rats $(280 \pm 20 \mathrm{~g})$, which were housed under standard laboratory conditions $\left(22 \pm 2{ }^{\circ} \mathrm{C}\right.$ room temperature; $55 \pm 5 \%$ room humidity; $12: 12 \mathrm{~h}$ light/dark cycle). Animals had free access to water and food and they were sacrificed $24 \mathrm{~h}$ after the last drug administration. All experiments were performed in accordance with the Czech act No. 246/1992 and with the approval of both the local and national Czech Central Commission for Animal Welfare.

In the Experiment $\mathrm{I}$, after 5 days of acclimatization, rats were randomly divided into four groups per 9-10 animals and were treated intragastrically with safranal dissolved in a mixture of propylene glycol and $5 \%$ glucose $(1: 1)$ at the doses of 4,20 , and $100 \mathrm{mg} / \mathrm{kg} /$ day. The administrations were repeated for 10 consecutive days. The control group was administered with appropriate volume of vehicle $(2 \mathrm{ml} / \mathrm{kg})$. Safranal was administered intragastrically to simulate the same route of administration of food supplements and due to its poor solubility in aqueous solvents and thus inability to prepare appropriate parenteral solution.

In the Experiment II, after 5 days of acclimatization, rats were randomly divided into four groups per 9-10 animals and were treated intraperitoneally with crocin dissolved in saline at the doses of 4 , 20 , and $100 \mathrm{mg} / \mathrm{kg} /$ day for 9 consecutive days. The control group was administered with appropriate volume of vehicle $(1 \mathrm{ml} / \mathrm{kg})$. Crocin was administered intraperitoneally due to its poor absorption through the gastrointestinal tract (Singla and Bhat 2011).

\section{Preparation of rat liver microsomes}

The RLM were isolated from 3 grams of liver tissue of individual animals by differential ultracentrifugation $(19000 \times \mathrm{g}$ and $105000 \times \mathrm{g})$ in $20 \mathrm{mM}$ Tris/ $\mathrm{KCl}$ buffer ( $\mathrm{pH}=7.4)$ including washing with $0.15 \mathrm{M} \mathrm{KCl}$ and finally diluted in $0.25 \mathrm{M}$ Tris/sucrose buffer $(\mathrm{pH}=7.4)$. The total protein content in the microsomal preparations was assessed according to Lowry et al. (1951) using bovine serum albumin as a standard. Determination of total CYP content was assessed using CO-difference spectroscopy method according to Omura et al. (1964).

Determination of cytochrome P450 activity in rat liver microsomes

Assessment of the activity of CYP2A, CYP2B, CYP2C11, and CYP3A enzymes was based on the rate of testosterone biotransformation in RLM with a NADPH generating system according to the previously described method of Wójcikowski et al. (2008) with a slight modification. The incubation mixture of final volume of $0.5 \mathrm{ml}$ contained phosphate buffer $(50 \mathrm{mM}$; $\mathrm{pH}=7.4)$, EDTA (1.1 mM), NADP (1.2 mM), glucose-6-phosphate (4.4 mM), $\quad \mathrm{MgCl}_{2} \quad$ (3.2 mM), glucose-6-phosphate dehydrogenase $(0.5 \mathrm{U}$ in $0.5 \mathrm{ml})$, RLM $(25 \mu \mathrm{l})$, and 
testosterone, which was added to be in the final concentration of $400 \mu \mathrm{M}$. The reaction was stopped after $15 \mathrm{~min}$ of incubation at $37^{\circ} \mathrm{C}$ by adding $50 \mu \mathrm{l}$ of methanol and by cooling down in ice.

Table 1. CYP specific metabolites of testosterone (modified from Wójcikowski et al. 2008, Kot and Daniel 2008, Chovan et al. 2007, Kobayashi et al. 2002).

\begin{tabular}{ll} 
CYP & Metabolites of testosterone \\
\hline $2 \mathrm{~A}$ & $7 \alpha$-hydroxytestosterone \\
$2 \mathrm{~B}$ & $16 \beta$-hydroxytestosterone \\
$2 \mathrm{C} 11$ & $2 \alpha$-hydroxytestosterone \\
$2 \mathrm{C} 11$ & $16 \alpha$-hydroxytestosterone \\
$3 \mathrm{~A}$ & $2 \beta$-hydroxytestosterone \\
$3 \mathrm{~A}$ & $6 \beta$-hydroxytestosterone \\
\hline
\end{tabular}

The concentrations of testosterone and its specific metabolites were measured by a HPLC system (Shimadzu LC-10) with the UV detector (Shimadzu SPDM10AVP) by the modified method of Haduch et al. (2006). After addition of the internal standard (prednisone), the analytes were extracted from the microsomal suspension with dichloromethane $(4 \mathrm{ml})$, and the residue obtained after evaporation of extracts was dissolved in $200 \mu \mathrm{l}$ of $50 \%$ methanol. An aliquot (45 $\mu \mathrm{l})$ was injected into the HPLC system, and the mobile phase was used in the following gradient mode: time 0 to

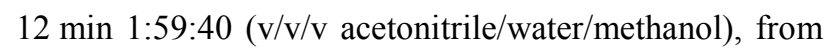
12:00 to $17: 50 \mathrm{~min} 1: 48: 51$ and from $17: 50$ to the end of the analysis in the 25:00 min 1:59:40. The flow rate was $0.8 \mathrm{ml} / \mathrm{min}$. Analytical column (Kinetex 2.6u PFP 100A, $150 \times 4.60 \mathrm{~mm}$ ) was purchased from Phenomenex (Torrance, CA, USA), and the absorbance was measured with DAD detector at the wavelength $245 \mathrm{~nm}$. Metabolic activities of all CYP enzymes were studied by measuring the rates of CYP specific reaction (Table 1) and expressed as the metabolite molar concentration/min/mg of total protein in RLM.

\section{Statistical analysis}

The results were statistically evaluated using the non-parametric Kruskal-Wallis test, and performed using the Statistica 12 software (StatSoft, Inc. 2013). Results were regarded as statistically significant when $\mathrm{p} \leq 0.05$.

\section{Results}

\section{Experiment I (safranal)}

The analysis revealed that the systemic administration of the highest dose $(100 \mathrm{mg} / \mathrm{kg})$ of safranal significantly increased the total protein content (149\% of the control group value) and also the total CYP content ( $151 \%$ of the control group value) (Fig. 1A). Other doses of safranal did not show any significant changes at the levels of the total protein and CYP content in RLM. However, both parameters were slightly elevated in comparison to the controls.

Changes in the metabolic activity were detected in all selected CYP enzymes except CYP2A (measured as a rate of $7 \alpha$-hydroxylation of testosterone) (Fig. 2). The metabolic activity of CYP2B (measured as a rate of the $16 \beta$-hydroxylation of testosterone) was significantly increased only at the highest dose of safranal. The effect of safranal on CYP3A is not convincing because the rate of $6 \beta$-hydroxylation was significantly increased, while $2 \beta$-hydroxylation of testosterone was not changed (both believed to reflect CYP3A metabolic activity). The lowest dose of safranal significantly increased the rate of $2 \alpha$ - and 16 $\alpha$-hydroxylation of testosterone, which is considered as CYP2C11 specific reaction.
(A) SAFRANAL

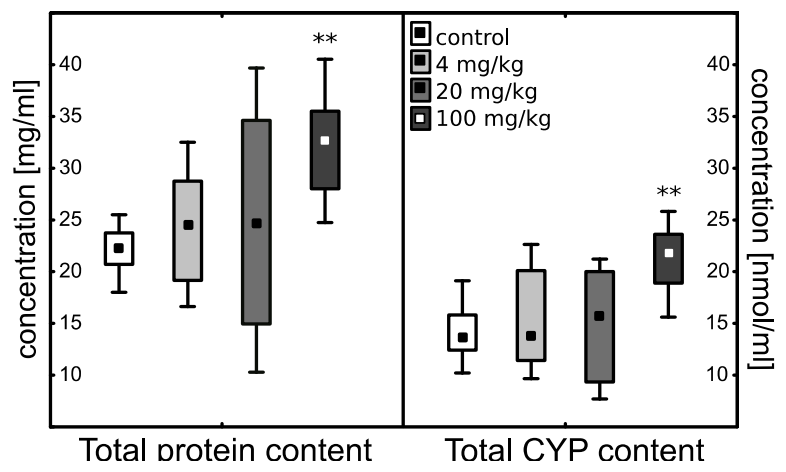

(B) CROCIN

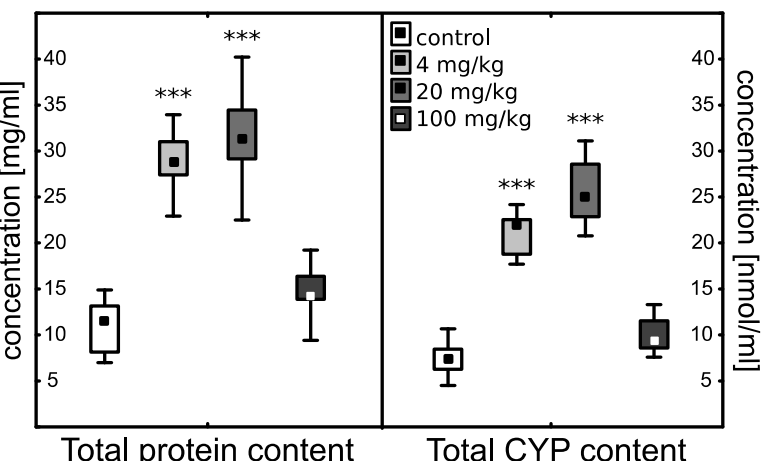

Fig. 1. The effect of systemic administration of safranal (A) and crocin (B) on the total protein and the total CYP content in RLM. All values are expressed as box plots with median (box $25 \%-75 \%$; whiskers Min-Max without outliers). Statistical significance with respect to the control group is indicated with $* * p \leq 0.01, * * * p \leq 0.001$. 
(A)

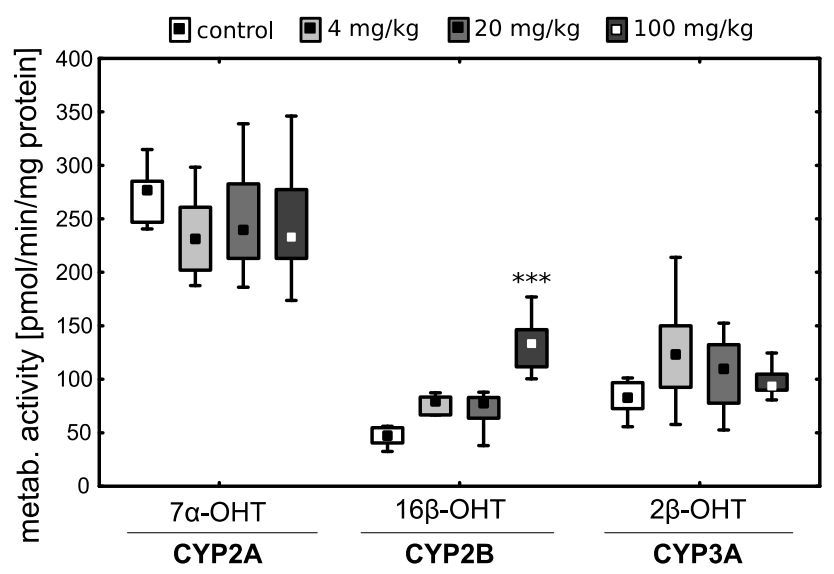

(B)

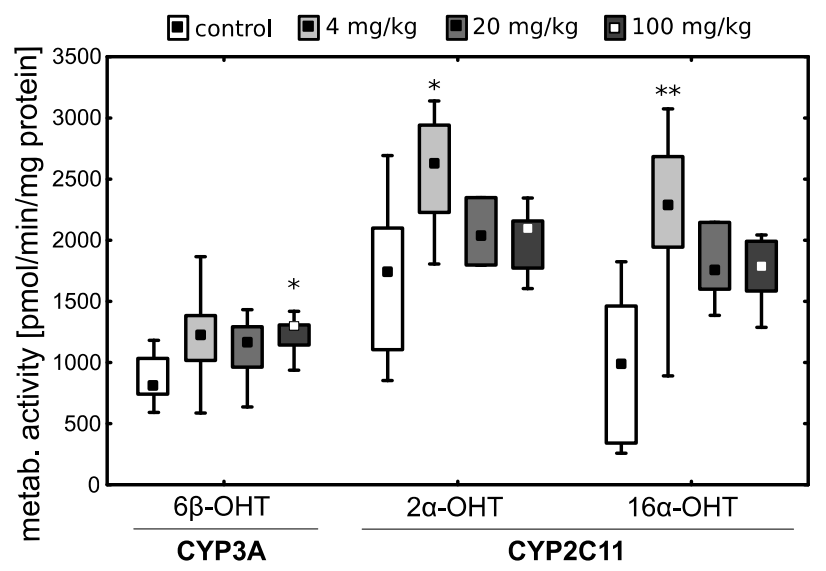

Fig. 2. The metabolic activity of selected CYP enzymes in RLM after safranal systemic administration, measured as the rate of testosterone hydroxylation. (A) The metabolic activity of CYP2A, CYP2B and CYP3A (2 3 -hydroxylation). (B) The metabolic activity of CYP3A (6 6 -hydroxylation) and CYP2C11. All values are expressed as box plots with median (box $25 \%-75 \%$; whiskers Min-Max without outliers). Statistical significance with respect to the control group is indicated with $* p \leq 0.05, * * p \leq 0.01, * * * p \leq 0.001$.

(A)

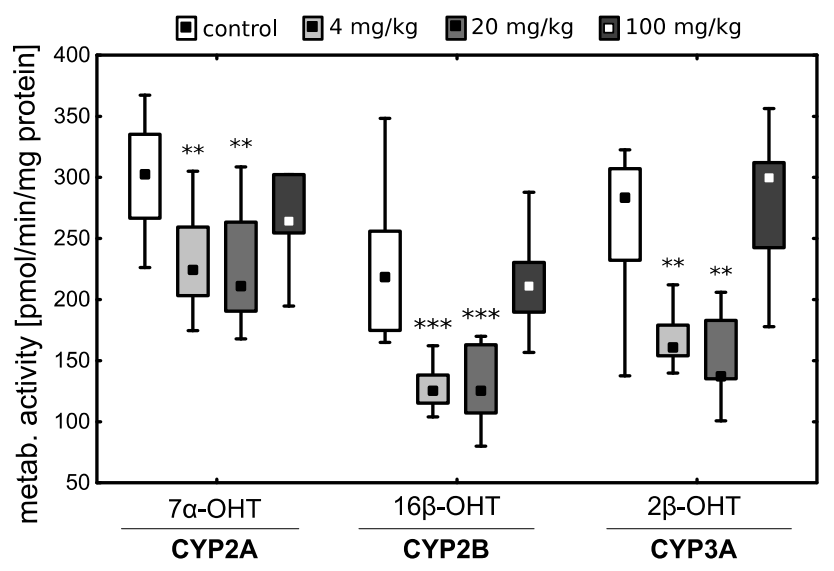

(B)

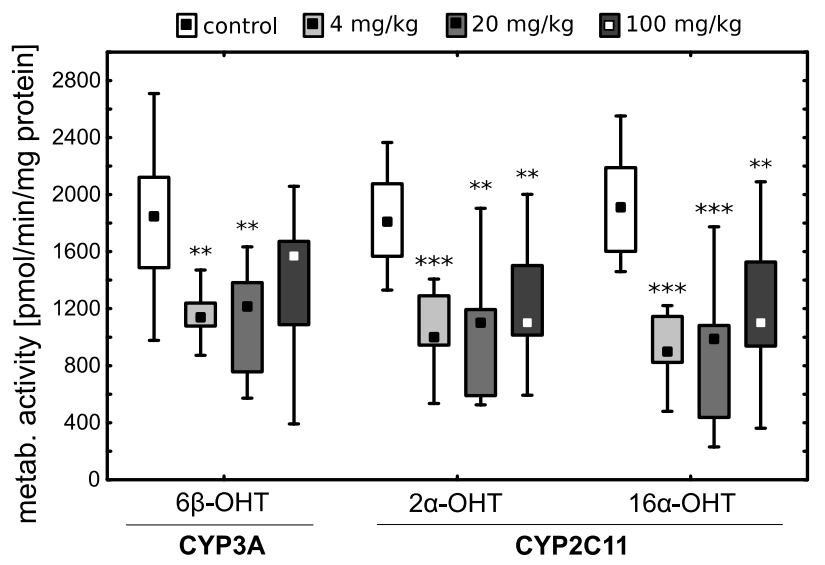

Fig. 3. The metabolic activity of selected CYP enzymes in RLM after crocin systemic administration, measured as a rate of testosterone hydroxylation. (A) The metabolic activity of CYP2A, CYP2B and CYP3A (2 $\beta$-hydroxylation). (B) The metabolic activity of CYP3A (6 $\beta$-hydroxylation) and CYP2C11. All values are expressed as box plots with median (box $25 \%-75 \%$; whiskers Min-Max without outliers). Statistical significance with respect to the control group is indicated with ${ }^{*} \mathrm{p} \leq 0.05, * * \mathrm{p} \leq 0.01, * * * \mathrm{p} \leq 0.001$.

\section{Experiment II (crocin)}

The obtained results showed that the systemic administration of crocin significantly increased the level of both the total protein and the total CYP content, at the doses $4 \mathrm{mg} / \mathrm{kg}$ (up to $259 \%$ and $280 \%$ of the control, respectively) and at $20 \mathrm{mg} / \mathrm{kg}$ (up to $278 \%$ and $343 \%$ of the control, respectively) (Fig. 1B). The amount of total protein and the total CYP content remained unchanged at the highest dose of crocin.

The administration of crocin, unlike safranal, caused reduction of the metabolic activity of selected CYP enzymes (Fig. 3). The determination of CYP2B metabolic activity revealed significant decrease at the doses of 4 and $20 \mathrm{mg} / \mathrm{kg}$ and the same result was observed in the metabolic activity of CYP2A. All doses of crocin significantly decreased the metabolic activity of CYP2C11. In the case of CYP3A, significant decrease was observed in the rate of both $6 \beta$-hydroxylation and $2 \beta$-hydroxylation of testosterone at the doses of 4 and $20 \mathrm{mg} / \mathrm{kg}$.

\section{Discussion}

Our results demonstrate the ability of systemic administration of safranal and crocin to increase the total protein and the total CYP content in RLM and to change the metabolic activity of different CYP enzymes. Thereby, tested substances could raise the risk of 
interactions with co-administered drugs metabolized by the same pathway. Several clinical trials confirmed that saffron may be of therapeutic benefit especially in the treatment of mild to moderate depression (Basti et al. 2007, Akhondzadeh et al. 2004, Moshiri et al. 2006). This is of interest because currently psychiatric patients with depression are treated by a wide range of synthetic antidepressants, but up to $30 \%$ of them are pharmacoresistant without a significant clinical improvement (Micale et al. 2013). Food supplements containing saffron extract are relatively safe, and so they are a viable alternative to the conventional drugs. The daily dose of saffron extract is $30 \mathrm{mg}$ according to clinical trials recommendations (Basti et al. 2007, Akhondzadeh et al. 2004, Moshiri et al. 2006).

To the best of our knowledge, no studies exist up to date dealing with the effects of safranal or crocin on CYP3A, CYP2C11, CYP2B, and CYP2A enzymes. However, it was described that metabolic activity of CYP enzymes is influenced by a large number of natural carotenoids, and the authors reported either decreased or increased CYP metabolic activity (Louisa et al. 2009, Jewell and O'Brien 1999, Satomi and Nishino 2013, Wang and Leung 2010). Our experiment showed that the metabolic activity of CYP2A, CYP2B, CYP2C11, and CYP3A was decreased after administration of carotenoid crocin. Safranal significantly increased the metabolic activity of CYP2B and CYP2C11, while the metabolic activity of CYP2A enzyme was without any significant changes. The effect of safranal on the testosterone hydroxylation via CYP3A was uncertain because $6 \beta$-hydroxylation was significantly increased, while $2 \beta$-hydroxylation of testosterone was not changed. Therefore, in our future experiments, we aim to use other probe substrates (nifedipine or midazolam) to determine the influence of safranal on CYP3A.

Interestingly, the total protein content was increased by safranal as well as by crocin. However, the metabolic activity of CYP2B, CYP2C11 and CYP3A enzymes was increased in the case of safranal and decreased in the case of crocin and CYP2A, CYP2B, CYP2C11, and CYP3A. The specific mechanism by which safranal or crocin influence CYP enzymes is unknown and we can therefore only hypothesize that this discrepancy could be explained by increase in CYP content including those CYP enzymes whose activity was not assessed in this study. Thus, we have observed apparent decrease in the CYP activity when the activity was calculated per mg of total protein or per nmol of total CYP. Other explanations could also exist, such as the negative feedback on reduced activity of main metabolic enzyme system. It is essential to carry out further experiments to confirm or reject these hypotheses.

To conclude, the obtained results showed that safranal and crocin influence the apparent metabolic activity of CYP enzymes in RLM. It would be necessary to take into account the risk of possible interactions with substances metabolized by CYP enzymes in the case of use of these substances in the clinical practice. This risk becomes more serious as food supplements containing saffron extracts are already available and their influence on the human CYP enzymes is not estimated yet. We report the ability of safranal and crocin to influence the activity at least of some rat liver CYP enzymes. Nevertheless, the daily intake of crocin and safranal from the food supplements is much lower in comparison to the doses administered to rats in our study and the substrate specificity of the rat CYP enzymes is not fully identical to human enzymes.

\section{Conflict of Interest}

There is no conflict of interest.

\section{Acknowledgements}

This work was supported by projects MUNI/A/1116/2014 and CZ.1.05/1.1.00/02.0068.

\section{References}

AKHONDZADEH S, FALLAH-POUR H, AFKHAM K, JAMSHIDI AH, KHALIGHI-CIGAROUDI F: Comparison of Crocus sativus L. and imipramine in the treatment of mild to moderate depression: a pilot double-blind randomized trial [ISRCTN45683816]. BMC Complement Altern Med 4: 12, 2004.

ALAVIZADEH SH, HOSSEINZADEH H: Bioactivity assessment and toxicity of crocin: a comprehensive review. Food Chem Toxicol 64: 65-80, 2014.

BASTI AA, MOSHIRI E, NOORBALA AA, JAMSHIDI AH, ABBASI SH, AKHONDZADEH S: Comparison of petal of Crocus sativus L. and fluoxetine in the treatment of depressed outpatients: a pilot double-blind randomized trial. Prog Neuropsychopharmacol Biol Psychiatry 31: 439-442, 2007. 
BATHAIE SZ, MOUSAVI SZ: New applications and mechanisms of action of saffron and its important ingredients. Crit Rev Food Sci Nutr 50: 761-786, 2010.

CHOVAN JP, RING SC, YU E, BALDINO JP: Cytochrome P450 probe substrate metabolism kinetics in Sprague Dawley rats. Xenobiotica 37: 459-473, 2007.

HADUCH A, WÓJCIKOWSKI J, DANIEL WA: The effect of tricyclic antidepressants, selective serotonin reuptake inhibitors (SSRIs) and newer antidepressant drugs on the activity and level of rat CYP3A. Eur Neuropsychopharmacol 16: 178-186, 2006.

HOSSEINZADEH H, NORAEI NB: Anxiolytic and hypnotic effect of Crocus sativus aqueous extract and its constituents, crocin and safranal, in mice. Phytother Res 23: 768-774, 2009.

JEWELL C, O'BRIEN NM: Effect of dietary supplementation with carotenoids on xenobiotic metabolizing enzymes in the liver, lung, kidney and small intestine of the rat. Br J Nutr 81: 235-242, 1999.

KOBAYASHI K, URASHIMA K, SHIMADA N, CHIBA K: Substrate specificity for rat cytochrome P450 (CYP) isoforms: screening with cDNA-expressed systems of the rat. Biochem Pharmacol 63: 889-896, 2002.

KOT M, DANIEL WA: The relative contribution of human cytochrome P450 isoforms to the four caffeine oxidation pathways: an in vitro comparative study with cDNA-expressed P450s including CYP2C isoforms. Biochem Pharmacol 76: 543-551, 2008.

LOUISA M, SUYATNA FD, SETIAWATI A, JUSMAN SWA: The effect of lycopene on the total cytochrome P450, CYP1A2 and CYP2E1. Med J Indonesia 18: 233-238, 2009.

LOWRY OH, ROSEBROUGH NJ, FARR AL, RANDALL RJ: Protein measurement with the folin phenol reagent. J Biol Chem 193: 265-275, 1951.

MELNYK JP, WANG S, MARCONE MF: Chemical and biological properties of the world's most expensive spice: saffron. Food Res Int 43: 1981-1989, 2010.

MICALE V, DI MARZO V, SULCOVA A, WOTJAK CT, DRAGO F: Endocannabinoid system and mood disorders: priming a target for new therapies. Pharmacol Ther 138: 18-37, 2013.

MOSHIRI E, BASTI AA, NOORBALA AA, JAMSHIDI AH, HESAMEDDIN ABBASI S, AKHONDZADEH S: Crocus sativus L. (petal) in the treatment of mild-to-moderate depression: a double-blind, randomized and placebocontrolled trial. Phytomedicine Int J Phytother Phytopharm 13: 607-611, 2006.

MOSHIRI M, VAHABZADEH M, HOSSEINZADEH H: Clinical applications of saffron (Crocus sativus) and its sonstituents: a review. Drug Res 65: 287-295, 2015.

NOORBALA AA, AKHONDZADEH S, TAHMACEBI-POUR N, JAMSHIDI AH: Hydro-alcoholic extract of Crocus sativus L. versus fluoxetine in the treatment of mild to moderate depression: a double-blind, randomized pilot trial. J Ethnopharmacol 97: 281-284, 2005.

OMURA T, SATO R: The carbon monoxide-binding pigment of liver microsomes I. Evidence for its hemoprotein nature. J Biol Chem 239: 2370-2378, 1964.

REZAEE R, HOSSEINZADEH H: Safranal: from an aromatic natural product to a rewarding pharmacological agent. Iran J Basic Med Sci 16: 12-26, 2013.

RÍOS JL, RECIO MC, GINER RM, MÁÑEZ S: An update review of saffron and its active constituents. Phytother Res 10: 189-193, 1996.

SATOMI Y, NISHINO H: Inhibition of the enzyme activity of cytochrome P450 1A1, 1A2 and 3A4 by fucoxanthin, a marine carotenoid. Oncol Lett 6: 860-864, 2013.

SCHMIDT M, BETTI G, HENSEL A: Saffron in phytotherapy: pharmacology and clinical uses. Wien Med Wochenschr 157: 315-319, 2007.

SINGLA RK, BHAT VG: Crocin: an overview. Indo Glob J Pharm Sci 4: 281-286, 2011.

TARANTILIS PA, TSOUPRAS G, POLISSIOU M: Determination of saffron (Crocus sativus L.) components in crude plant extract using high-performance liquid chromatography-UV-visible photodiode-array detection-mass spectrometry. J Chromatogr A 699: 107-118, 1995.

WANG H, LEUNG LK: The carotenoid lycopene differentially regulates phase I and II enzymes in dimethylbenz[a]anthracene-induced MCF-7 cells. Nutrition 26: 1181-1187, 2010.

WINTERHALTER P, STRAUBINGER M: Saffron - renewed interest in an ancient spice. Food Rev Int 16: 39-59, 2000.

WÓJCIKOWSKI J, GOŁEMBIOWSKA K, DANIEL WA: Regulation of liver cytochrome P450 by activation of brain dopaminergic system: physiological and pharmacological implications. Biochem Pharmacol 76: 258-267, 2008. 